\title{
Nursery Cultural Practices and Morphological Attributes of Longleaf Pine Bare-Root Stock as Indicators of Early Field Performance
}

\author{
Athens, Georgla \\ and \\ H. David Muse, Professor \\ Department of Mathematics \\ University of North Alabama \\ Florence, Alabama
}

Glyndon E. Hatchell, Research Forester (Retired)

Institute for Mycorrhizal Research and Development

\begin{abstract}
Seven morphological characteristics of 1-0 longleaf pine (Pinus palustris Mill.) stock were evaluated on a total of 1,600 seedlings representing eight combinations of nursery cultural treatments. Seedlings were outplanted completely at random, with respect to rows and planting positions, on a deep sandy site in South Carolina. Field performance of individual seedlings was evaluated after two growing seasons that were extremely dry. Statistical tests were derived for determining differences in field performance of groups of seedlings, both within and between treatments. Groups of seedlings that received vertical root-pruning treatment (sidecutting) in the nursery and possessed key attributes-either 14 or more strong, first-order lateral roots for a low-fibrosity root system or a minimum of 6 strong laterals for medium- or high-fibrosity root system-met the preset goal of 80 percent survival and 60 percent of planting stock in active height growth after two growing seasons. Within a pruning treatment, all seedlings possessing these key morphological attributes, regardless of root-collar diameters, performed equally well in the field. For each of the three fibrous-root ratings, seedlings that received vertical root pruning in nursery beds had significantly higher survival than unpruned seedlings. Vertical root pruning also significantly increased the proportion of planting stock in active height growth after the second growing season.
\end{abstract}

Keywords: Seedling quality, morphological grades, vertical root pruning, Pinus palustris. 